\title{
Detection and Classification of Cotton Leaf Diseases Using Faster R-CNN on Field Condition Images
}

\section{Rehan Sarwar $^{1 *}$, Muhammad Aslam ${ }^{1}$, Khaldoon S Khurshid ${ }^{1}$, Tauqir Ahmed $^{1}$, Ana Maria Martinez-Enriquez ${ }^{2}$ and Talha Waheed ${ }^{1}$}

${ }^{1}$ Department of Computer Science, University of Engineering and Technology,

Lahore, Pakistan

${ }^{2}$ Department of Computer Science, Center of Investigations and Advanced Studies (CINVESTAV-IPN), Mexico City, Mexico

*Corresponding Author: Rehan Sarwar, Department of Computer Science, University of Engineering and Technology, Lahore, Pakistan.
Received: July 28, 2021

Published: September 24, 2021

(C) All rights are reserved by Rehan Sarwar., et al.

\begin{abstract}
Disease's classification of the cotton leaf can increase the cotton yield. Deep learning is emerging as a powerful method in many fields, and much research has been done in Agriculture in real-time detection of cotton leaf diseases. Convolution Neural Networks have played a vital role in plant classification and identification of diseases, but still, work is needed to help farmers and pathologists correctly detect and classify diseases. Manual checking of disease crops takes a lot of time and cost, and it is hectic. Besides, the wrong diagnosis entails inaccurate conclusions, treatment, and significant expense. This paper proposes to train a deep learning Faster R-CNN model on cotton crop leaf dataset (CCLDataset) for detecting and classifying diseases on leaves, including both healthy and diseases. Plant Village dataset is the reference in finding the best feature extractor from VGG-16, InceptionV1, and V2. Additionally, it is a base model in Faster R-CNN. Transfer learning is performed on CCLDataset when trained on the model Faster R-CNN inceptionV2 coco by replacing the output layers of coco with CCLDataset to detect and classify leaf diseases. The experimental results show a mean average precision (mAP) of $87.1 \%$.
\end{abstract}

Keywords: Cotton Leaf Diseases; Object Detection; Faster R-CNN; CCLDataset; Mean Average Precision

\section{Abbreviations}

mAP: Mean Average Precision; CCLDataset: Cotton Crop Leaf Dataset; CNN: Convolutional Neural Networks.

\section{Introduction}

In cotton-producing countries, Pakistan is ranked fourth worldwide [1]. Along with the Indus River irrigation system, cotton cultivation is nearly 3 million/ha and is recognized as the backbone of the economy of the country. In many developing countries, cotton, also referred to as "White Gold", is an important crop. The yield of the cotton crop is dependent on the management of individuals and the environment where it grows. For many years there is a decline in cotton production, and yield is stagnant. Cotton production in 2018-2019 stood at 9.8 million bales, more minor than the production during 2017-2018, which stood at 11.9 million bales. A sharp decline of 17.5 percent in the yield during 2018-2019 over the previous year's yield due to diseases, unfavorable weather condition and water availability [2]. Cotton production further drops by $6.9 \%$ during 2019-2020 from the previous year and estimated to be around 9.178 million bales [3]. Multiple factors are responsible for the decline in cotton production, including heavy rain during sowing, a decline in cotton crop area due to harvesting of late wheat, soil, weather, pest, and diseases. Multiple social and economic factors directly affect the cotton yield like illiterate farmers, 
lack of guidance, small lands, inability to adopt innovations, high cost, and insecurity [4]. Plant disease is one of the leading causes of the decline in production. Nearly $50 \%$ reduction in yield each year is due to diseases. Plant diseases diagnosis through naked-eye observation of symptoms incorporates a high complexity. Even experienced pathologists often fail to diagnose diseases which leads to wrong conclusions and treatments. Cultivating cotton is a type of extensive farming which excessively requires resource utilization to protect the crop from pests. Multiple factors are responsible for the low yield of cotton, but the top factor in reducing the quality and quantity of cotton crop are insects. Climate factors, such as temperature, rainfall, and relative humidity, also play a crucial role in the population dynamics of insect pests [5].

Exploring the possibility of acquiring good results in the cotton crop and improving yield, we used deep learning with proven efficacy and methods and techniques to recover essential data from image processing, segmentation, and others. For instance, by accessing some common properties, it is possible to identify the type of plant, the disease on a leaf by accessing common properties like color, texture, and shape. Computer vision could not help plant disease classification due to pixels of images being low and semantics perceived by users being high. In solving computer vision problems, machine learning has proved useful out of many different techniques. For the development of intelligent systems, machine learning shows significant potential in the prediction and classification of a large dataset as well as solving complex problems [6].

For Computer vision, understanding agriculture is still a difficult task. Experts in machine learning are required to outline which features would be best for problem using machine learning algorithms. For this, Convolutional Neural Networks (CNN) is used to extract the suitable features to solve classification problem automatically. For image analysis $\mathrm{CNN}$ is an appropriate technique. CNN's have several layers, and every layer is extracting some features. Starting or Initial layers learn simple features like edges and colors. Later layers in the network learn complex features related to the classification problem and at the end the extracted feature from the last layer is fed into Neural Network for classification [7]. Our research aims to detect and classify cotton leaf diseases using object detection on real-time field condition images of cotton leaves to help farmers and learners diagnose the disease correctly. Faster R-CNN is applied with inceptionV2 as a base model.
This paper's organization is as follows: Section 2 describes the related work for plant leaf diseases classification and identification. Section 3 presents the methodology and approach. Section 4 exposes the materials and methods followed in our research. Section 5 discusses the obtained results of the experimentation and the used metrics. Finally, the Conclusion section draws our contributions and explains future work.

\section{Related work}

In the past years, different works have been done to classify cotton leaf images and detection of diseases using machine learning techniques. In 2015, different works were done to classify cotton leaf diseases using different pattern recognition methods for classification and identification of leaf diseases on cotton. Rothe PR and Kshirsagar RV proposed a pattern recognition system for three cotton leaf diseases identification and classification namely Alternaria, Myrothecium and Bacterial blight. This cotton dataset was collected using two different Cannon digital camera A460 and EOS550D from the cotton fields in natural condition. To remove noise in the images, preprocessing was done by applying Gaussian filter and image segmentation was done by using active contour model. Different Hu moments features were extracted after image preprocessing and segmentation. Adaptive neuro fuzzy was used to train dataset for the classification of cotton leaf diseases and gave an accuracy of $85 \%$ [8].

In 2016 Patki SS and Sable GS did classification using Multi SVM on cotton leaf diseases. Three different cotton leaf diseases of White Spot, Red Spot and Crumple Leaf was collected using high resolution camera and stored in the format of jpg. Collected dataset was made up of four different classes which contained three cotton leaf diseases and one healthy leaf class of 103 sample images with black background. These images were preprocessed by cropping and resizing into a standard size. These resized RGB images were then converted to different color space of YCbCr format which gave chrominance and luminance information. In order to suppress the unwanted part image segmentation was applied to separate leaf and disease part of the image from the background by using Otsu's thresholding method. Texture and color features from the segmented images were extracted and Multi SVM classifier was used as a classification technique for diseases classification which gave an accuracy of $87.5 \%$ [9]. 
Sarangdhar AA and Pawar VR in 2017 proposed a system for classification and identification of five different cotton leaf diseases using SVM based regression system. Digital camera was used to collect RGB images of five different cotton leaf diseases. A sample of 900 images were collected and saved in jpg format. For training and testing purpose 629 images were trained and for testing $271 \mathrm{im}$ ages were used. Different image preprocessing methods were used for the reduction of noises which include resizing of images to 250 $x 250$, gabor and median filtering to get good quality images. For the extraction of lesion regions in the images, segmentation was applied. Region of interest was extracted by applying thresholding and color transformation. RGB images were transformed to $\mathrm{YCbCr}$ format and bi-level thresholding was applied. Using partial least square regression, eight texture and color features were extracted from which two were texture and six were color. SVM based regression was applied for the classification of cotton leaf diseases after extraction of features. The system gave an accuracy of 83.26\% [10].

In 2018 Dubey YK., et al. did the classification of cotton leaf diseases by proposing a computer vision-based algorithm for the detection of diseases on the leaves by classifying them according to the class which it belonged to. Dataset was collected from online resources and contained four classes which include Alternaria, Bacterial Blight, White Flies and healthy leaves of laboratory samples of 20 images for each class. Images were preprocessed by different methods which include histogram, histon, roughness measure and peak selection in order to obtain region of interest. For the segmentation part superpixel segmentation was applied, which divide images into multiple segments of superpixel. Most algorithms produce unbalanced superpixel with different sizes and shape. To counter this problem SLIC algorithm was used for clustering pixels in the image and color space to obtain uniform and compact superpixels. Gray level co-occurrence matrix (GLCM) texture features were extracted in the feature extraction phase for the classification. A multi class SVM classifier was used for the classification of diseases which gave an average accuracy of $94 \%$ for the four classes [11].

In 2019 Kumari CU., et al. detected diseases on cotton leaves by extracting features using K-means clustering and did classification with artificial neural network (ANN). Cotton leaf spot and healthy images were acquired from PlantVillage dataset in the laboratory condition of size 250 x 250 . Image segmentation was done using
K-means clustering by separating the disease spot part and healthy leaf region. RGB images were loaded into MATLAB and were converted in $\mathrm{L}^{*} \mathrm{a}^{*} \mathrm{~b}^{*}$ color space where $\mathrm{L}$ signifies lightness, $\mathrm{a}$ and $\mathrm{b}$ represented chromaticity. Each pixel was reallocated to the nearest cluster and consist of three different segments of leaf images. Features were extracted from the segmented clusters. Four different steps were performed in feature extraction stage like entering only disease affected part of the leaf, convert it to gray scale, create Gray Level Co-occurrence Matrices and deriving features from GLCM. These resultant features include correlation, contrast, homogeneity, energy, mean, variance and standard deviation. These features were computed and given as an input for further processing. In the classification process these seven computed features were given to the neural network as input and results were extracted by training the neural network. ANN gave an average accuracy of $85 \%$ for bacterial leaf spot and targeted spot [12].

In 2020 Khairnar K. and Goje N. did diagnosis and disease detection on cotton leaf images via image processing-based approach. Firstly, images were attained using a digital camera and resized to $150 \times 150$ in the preprocessing phase. Images were then manually separated into different classes which include Bacterial Blight, Cercospora and Alternaria. RGB images were converted to $\mathrm{L}^{*} \mathrm{a}^{*} \mathrm{~b}^{*}$ color space. After this segmentation was done on the disease part using K-mean clustering. In the feature extraction stage color features were extracted using color co-occurrence matrix which include variance, expectancy and skewness. Texture features were extracted using GLCM which include entropy, energy, homogeneity, symmetry and contrast. For classification purpose SVM and ANN classifiers were used. SVM produced better result than ANN by obtaining an accuracy of $94 \%$ [13].

In 2021 Patil B. and Barkpalli V. gave a view of cotton healthy and unhealthy leaf image classification using different machine learning algorithms with WEKA. Under controlled conditions cotton leaf dataset was created by taking images in the field with natural background. This dataset contained nearly 3000 images of two different classes healthy and unhealthy leaves for training and testing. Modified factorization-based active contour was used for image segmentation. Two different features based on texture and color were extracted from segmented images which were used for training and testing by different machine learning algorithms like RF, NB, SVM, Ada Boost, KNN and MP. Performance of classifiers 
were better with color feature to classify healthy and unhealthy leaves. WEKA was used for the analysis of different classifiers by feeding the same features. Multilayer perceptron gave classification performance of $96.69 \%$ from other classifiers [14].

Caldeira RF., et al. used deep learning to identify lesions or damages on cotton leaf images. 60,659 images were periodically collected from cotton plantation during 2018 and 2019. This dataset contained both healthy and lesioned leaves with backgrounds and were resized to $102 \times 102$. After acquisition and pre-processing, images were fed into pipeline for different deep learning models like GoogleNet and ResNet50. Overall results achieved by GoogleNet and ResNet50 were $86.6 \%$ and $89.9 \%$ which were compared with traditional machine learning approaches by achieving overall accuracy above 70\% like Support Vector Machine, K-Nearest Neighbor, Artificial Neural Network and Neuro-Fuzzy. Convolutional Neural Network proved $25 \%$ precise from traditional machine learning algorithms when compared with each other [15]. Table 1 gives a brief overview of different authors involvement for cotton leaf diseases classification.

\begin{tabular}{|c|c|c|c|c|}
\hline Year & Author & $\begin{array}{c}\text { Pre- } \\
\text { processing }\end{array}$ & $\begin{array}{l}\text { Segmenta- } \\
\text { tion }\end{array}$ & Classifier \\
\hline 2015 & $\begin{array}{c}\text { Rothe PR and } \\
\text { Kshirsagar } \\
\text { RV }\end{array}$ & $\begin{array}{l}\text { Gaussian } \\
\text { filter }\end{array}$ & $\begin{array}{l}\text { Active con- } \\
\text { tour model }\end{array}$ & $\begin{array}{c}\text { Adaptive } \\
\text { neuro fuzzy }\end{array}$ \\
\hline 2016 & $\begin{array}{c}\text { Patki SS and } \\
\text { Sable GS }\end{array}$ & $\begin{array}{c}\text { RGB to } \mathrm{YCbCr} \\
\text { format }\end{array}$ & $\begin{array}{l}\text { Otsu's thresh- } \\
\text { olding }\end{array}$ & $\begin{array}{c}\text { Multi Class } \\
\text { SVM }\end{array}$ \\
\hline 2017 & $\begin{array}{c}\text { Sarangdhar } \\
\text { AA and Pawar } \\
\text { VR }\end{array}$ & $\begin{array}{c}\text { Gabor filter } \\
\text { and median } \\
\text { filter }\end{array}$ & $\begin{array}{l}\text { Color trans- } \\
\text { formation }\end{array}$ & SVM \\
\hline 2018 & $\begin{array}{c}\text { Dubey YK., } \\
\text { et al. }\end{array}$ & $\mathrm{N} / \mathrm{A}$ & Superpixel & $\begin{array}{c}\text { Multi Class } \\
\text { SVM }\end{array}$ \\
\hline 2019 & $\begin{array}{c}\text { Kumari CU., } \\
\text { et al. }\end{array}$ & $\mathrm{N} / \mathrm{A}$ & $\begin{array}{l}\text { K-means } \\
\text { clustering }\end{array}$ & ANN \\
\hline 2020 & $\begin{array}{l}\text { Khairnar K } \\
\text { and Goje N }\end{array}$ & $\begin{array}{c}\text { RGB to } L^{*} a^{*} b^{*} \\
\text { format }\end{array}$ & $\begin{array}{l}\text { K-means } \\
\text { clustering }\end{array}$ & SVM \\
\hline 2021 & $\begin{array}{l}\text { Patil B. and } \\
\text { Barkpalli V. }\end{array}$ & $\begin{array}{l}\text { Gaussian } \\
\text { filter }\end{array}$ & $\begin{array}{c}\text { Modified } \\
\text { factorization- } \\
\text { based active } \\
\text { contour }\end{array}$ & $\begin{array}{c}\text { MP, SVM, NB, } \\
\text { RF, KNN, Ada } \\
\text { Boost }\end{array}$ \\
\hline 2021 & $\begin{array}{l}\text { Caldeira RF., } \\
\text { et al. }\end{array}$ & \begin{tabular}{|c|} 
Manual \\
selection and \\
grouping \\
\end{tabular} & $\mathrm{N} / \mathrm{A}$ & $\begin{array}{c}\text { Google Net, } \\
\text { ResNet50 }\end{array}$ \\
\hline
\end{tabular}

Table 1: Literature survey review.
Methodology

The proposed system for leaf disease detection and classification problems accepts input in images represented by a threedimensional matrix. In Data pre-processing phase, images are resized and annotated, and pre-processed images are passed through the model. The model trains on the CCLDataset using an object detection model faster R-CNN with inceptionV2 as a base model on cotton leaves' field condition images. After training, evaluation is done on the dataset. In the evaluation process, data is validated. If an evaluation is not correct, the parameters are fine-tuned, and training is done again until it gets better and passed to the classifier. The model classifies the labels for the leaves on which detection and classification are done. The proposed system methodology is shown in figure 1.



Figure 1: Methodology.

The proposed methodology consists of three main steps:

- $\quad$ Fine-tuned different models like VGG-16, Inception V1, and V2 on the PlantVillage dataset to find the best feature extractor, which would be used as a base model in training our original dataset.

- $\quad$ Fine-tuned the coco pre-trained model of Faster R-CNN InceptionV2 on CCLDataset to detect and classify leaf diseases.

- Validation of model on the validation set of CCLDataset and subjective accuracy calculated from inference graph on the unseen images passed in the model. 


\section{Material and Methods}

\section{Dataset collection}

From July to August in 2019, cotton leaf disease images are collected from the cotton fields in Bahawalpur and Multan in Pakistan's natural environment. This cotton is naturally grown cotton by farmers and is not Bt transgenic cotton (genetically modified cotton plant). The images are captured sometime before the harvesting during the flowering and boll stage. To get the best results, images are collected on different days, including cloudy and sunny days. The dataset of cotton collected is organized into four classes and contained a total of $750+$ images. The classes contained different images of cotton leaf diseases as well as healthy leaves. Figure 2 shows CCLDataset sample images.
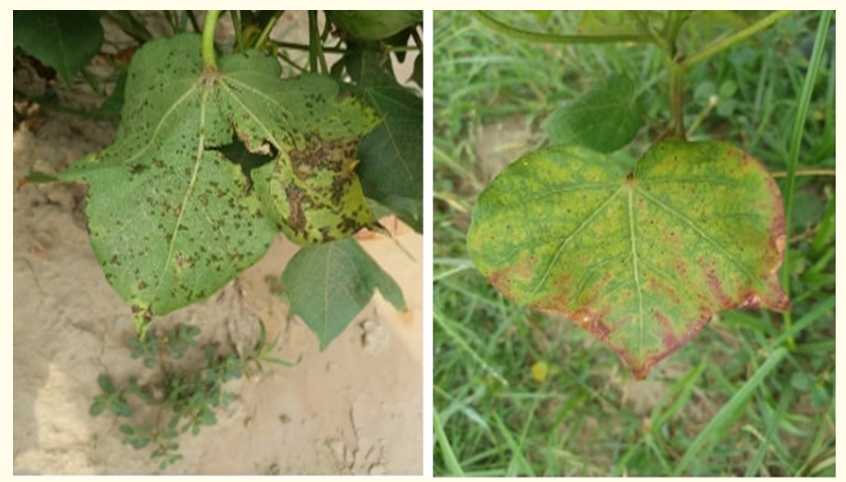

Figure 2: CCLDataset images.

To find the best feature extractor that can be used as a base model when object detection is performed on CCLDataset, another dataset named PlantVillage is used, which has 38 classes and $53,925+$ images of laboratory samples of size $256 \times 256$.

\section{Image preprocessing}

Image preprocessing includes two steps, resizing of images and annotations. First, images of cotton leaves are collected using three different Samsung mobiles. All the images have different dimensions and are resized to $720 \times 480$ for training and validation of the model. Secondly, for the annotation part, resized images are annotated manually according to their class. Rectangular boxes are drawn on the disease organized into four classes: Bacterial Blight, Mosaic, Pesticide burn, and healthy leaf.
Model

The CNN architecture applied in this work to detect and classify cotton leaf diseases is Faster R-CNN. Faster R-CNN is an object detection algorithm [16]. This model with training and testing methods is implemented using TensorFlow deep learning framework. NVIDIA GTX1080 TI card is used to implement training algorithms, using CUDA programming platform in Ubuntu 16.04 LTS operating system (Linux). Faster R-CNN is a combination of two modules; one is a fully convolutional network which proposes regions, and the other one is a Fast R-CNN detector that uses proposed regions. In R-CNN, networks mainly consist of "bounding boxes" generated by the regional proposal algorithm (RPA) and by locating objects in the image. Using a CNN, object features are obtained in a feature generation stage. Classification layers are used to predict the object belonging to a class. Also, to precisely make the coordinates of the bounding box object, a regression layer is used. The stand-alone part of the network in Fast R-CNN is the regional proposal algorithm. It takes roundabout 2 seconds/image for selective search algorithm (SSA), which runs on the CPU computation. Faster R-CNN uses additional convolutional network in order to produce the region proposals. This reduces time from $2 \mathrm{~s}$ to $10 \mathrm{~ms}$ per image in the region proposal and shares layers with the detection stages to improve feature representation. It is also referred to as a detection pipeline that uses Fast R-CNN, a detector network and RPN as RPA. GPU is used for the implementation of the training algorithm. The model Faster R-CNN is used with inceptionV2 as a base model for the detection and classification of cotton leaf diseases. Coco Pretrained faster R-CNN_inceptionV2 model is used by freezing and replacing the output layers with our CCLDataset containing four classes. This transfer learning process prevents us from training the model from scratch when the dataset's quantity is low. Usually, we express transfer learning through pre-trained models in computer vision [17]. As our local dataset is low in quantity, transfer learning helped train the CCLDataset, collected in the natural realfield conditions. The original loss function of faster R-CNN can be seen in Equation 1.

Where is probability predicted of anchor being an object. is ground-truth label, 1 is positive and 0 is negative anchor. stores predicted bounding box 4 coordinates while is related to positive anchor ground-truth box. Classification loss represented by and regression loss by and their normalized terms with, and as a balancing weight. 
Training and testing dataset

CCLDataset contained 750+ images and four classes. This dataset is divided into two parts, i.e., training and testing set. CCLDataset is split into an $80-20$ ratio for using $80 \%$ data in the training phase and the remaining $20 \%$ in the testing phase. 602 images were used in the training set, and 151 used in the testing set. Table 2 shows dataset splitting.

\begin{tabular}{|c|c|c|}
\hline Class & Training & Testing \\
\hline Bacterial Blight & 113 & 29 \\
\hline Mosaic & 184 & 45 \\
\hline Pesticide Burn & 172 & 43 \\
\hline Healthy & 133 & 34 \\
\hline Total & 602 & 151 \\
\hline
\end{tabular}

Table 2: CCL Dataset Splitting.

\section{Results and Discussion}

\section{Loss graph and Learning rate}

The result shown in this section is related to training of both the PlantVillage dataset and CCLDataset. PlantVillage dataset is used as a reference to find the best feature extractor. For this, different base models like VGG-16, InceptionV1 and V2 are tested. InceptionV2 extracted the best features and is used as a base model for object detection in Faster R-CNN. As the model is pre-trained on coco, transfer learning is applied on the CCLDataset by freezing the output layers in the faster R-CNN inceptionv2 coco model and applying our own dataset four classes, namely bacterial blight, mosaic, pesticide burn, and healthy leaves. When the CNN network is trained, the network becomes capable of learning features from the dataset. As CCLDataset is small and gets better results, we first used the PlantVillage dataset, which is similar to CCLDataset but contains fruits and vegetables healthy and disease leaves for reference to find the best feature extractor that can be used as a base model in our faster R-CNN object detection. The loss graph of PlantVillage dataset after 2000 epochs shown in figure 3. SGD optimizer is used to optimize the loss. The loss calculated for PlantVillage is 1.012. As we can see, without smoothing the graph in tensorboard, the loss is high in the starting phase, and it gradually decreases with every epoch.

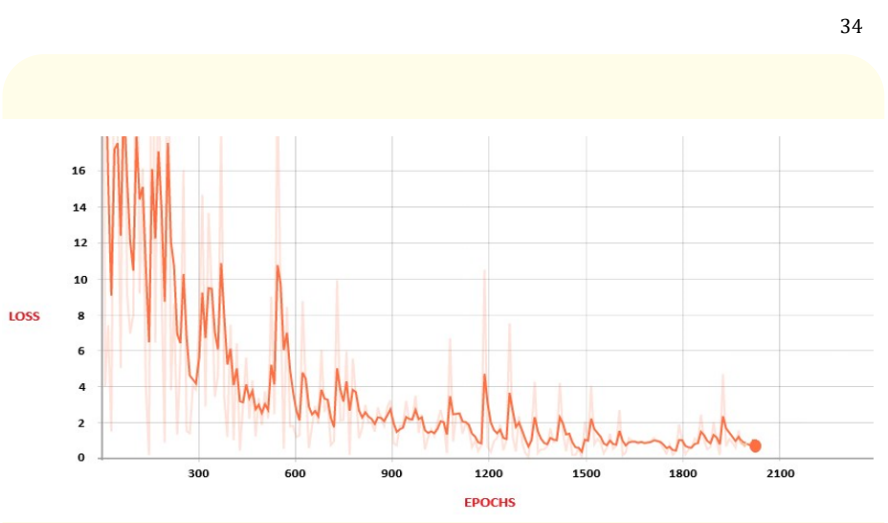

Figure 3: Loss graph of PlantVillage dataset.

After finding the best feature extractor from a similar dataset, we trained our CCLDataset using faster R-CNN object detection. Faster R-CNN inceptionV2 coco is used to train CCLDataset, which is pre-trained on the coco dataset containing 80 classes. When CCLDataset is trained, the coco dataset's output layers are replaced by the four classes of the CCLDataset and are trained on it. The number of steps for the training of CCLDataset for the detection and classification of the CCLDataset is $150 \mathrm{k}$, shown in figure 4. SGD optimizer is used to adjust the loss function when CCLDataset is trained. The overall loss on this dataset after training and testing is 0.01275 .

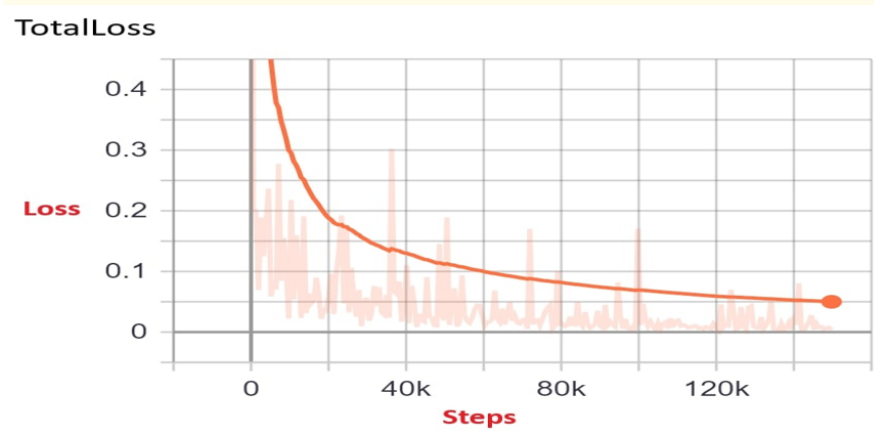

Figure 4: Loss graph of CCLDataset.

The learning rate for the CCLDataset is set to 0.0002. A lower learning rate is used to learn the features more optimally, resulting in longer training time and better results. As the range of the 
learning rate is between 0.0 to 1.0 . The learning rate for the CCLDataset is initially set at 0.0002 . At $90 \mathrm{k}$ steps learning rate decays to 0.00002 , and at $120 \mathrm{k}$ steps, it decays to 0.000002 . The value for SGD momentum is set to 0.9 with a batch size of 1 . Table 3 shows a loss for both datasets when trained.

\begin{tabular}{|c|c|c|}
\hline Dataset & Epochs/Steps & Loss \\
\hline Plant Village & $2 \mathrm{k}$ & 1.012 \\
\hline CCL Dataset & $150 \mathrm{k}$ & 0.0127 \\
\hline
\end{tabular}

Table 3: Final Epochs/Steps and Loss.

Image-based detection results

A different sample of images can be shown in the result section when passed through the model to detect and classify cotton leaf to be healthy or diseased. These results are generated when the dataset is trained on the faster R-CNN inceptionV2 coco. Transfer learning is used by replacing the output layers of the coco from the model with CCLDataset. The loss is calculated on CCLDataset, which contained four classes of cotton leaves, from which three of them are diseases, while one class contained healthy leaves. When unseen images passed through the model, the Faster R-CNN object detection determines and classifies the cotton leaves' diseases based on the classes it trained on. We discuss different resulting images with classes having either diseases or healthy leaves. Firstly, the resulting images without non-max suppression. As we can see, without suppression, different bounding boxes emerges with a different confidence score. Figure 5 shows the resulting images with three bounding boxes. Each bounding box is within another bounding box with a different confidence score without non-max suppression.

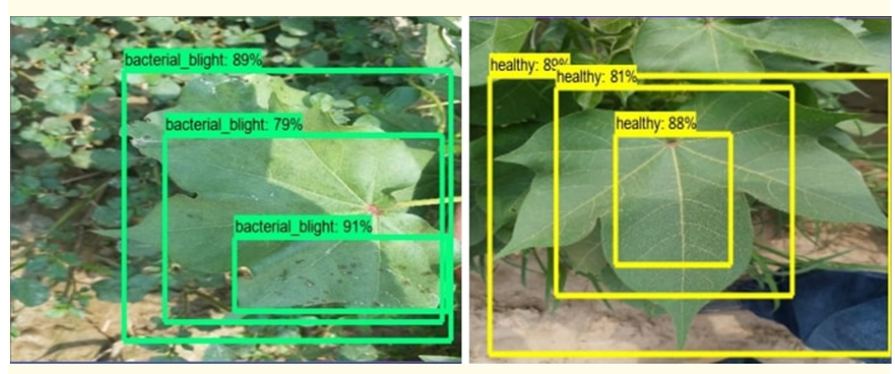

Figure 5: Without non-max suppression.
To acquire the best bounding box or suppress the other boxes to show a single box for an object, non-max suppression is used. Nonmax suppression works by selecting the best bounding box with the highest confidence score or removing or suppressing the boxes below the score threshold. This helped us find the best bounding box for the images when tested using our object detection model. Figure 6 shows the images on which non-max suppression is applied.

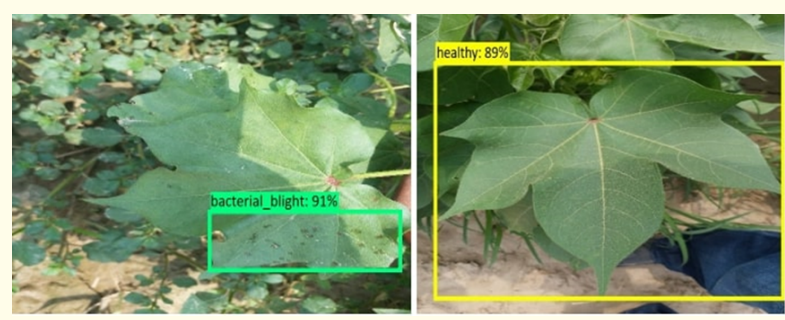

Figure 6: Non-max suppression.

As object detection accuracy cannot be calculated, we calculated a subjective accuracy using the inference graph by passing 40 unseen images in the model trained on four different classes of the CCLDataset. Subjective accuracy is calculated by checking how many images are correctly recognized by the model when passed. Out of 40 images, 36 images are correctly recognized by the model, and the subjective accuracy is calculated to be $90 \%$. When an object detection model is trained, evaluation metric does not have accuracy like a simple classification would have. Figure 7 shows the validated images results.

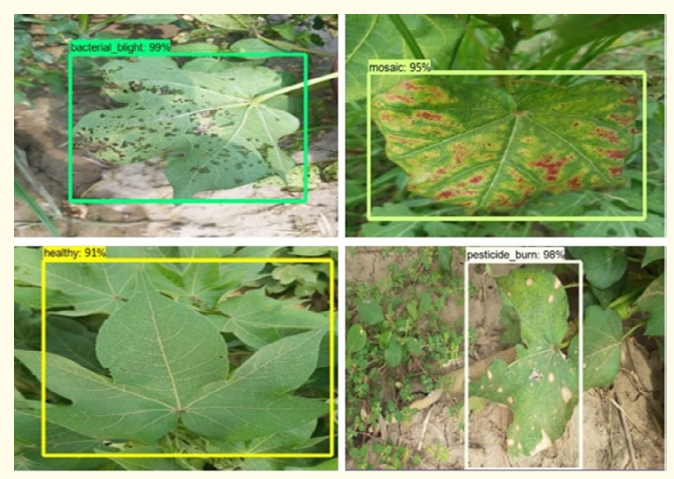

Figure 7: Validated images result through inference graph. 
While some images were wrongly identified or contained two different classes of bounding boxes when an image is passed from the model. Diseases and weather conditions have a great impact on the yield of crops. Experts regularly check the field to detect diseases on the crop. However, not all farmers can afford or rely on them as naked eye observation tends to lead to mistaken observation, and low yields are procured. To increase the yield and correctly identify the leaf diseases of cotton, deep learning algorithms for object detection are used to train on CCLDataset. Figures 5, 6 and 7 show results obtained by the system for the trained images. This system can support different individuals by correctly diagnosing leaf diseases and reducing human error to identify leaf diseases. As this crop is harvested for a limited time, the system can support correctly by identifying diseases on leaves.

The evaluation metric used for object detection in this research work is the mean average precision (mAP), which calculates precision for the entire model rather than calculating individual objects' precision. The mean average precision is 0.871 (87.1\%), shown in figure 8.

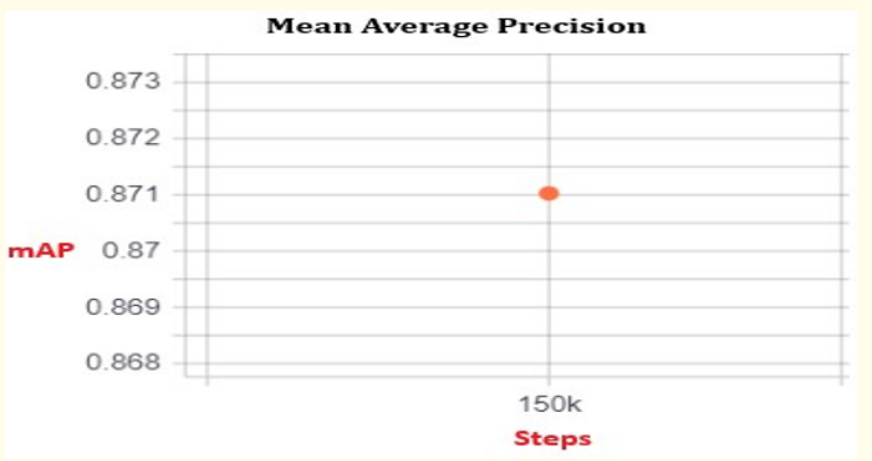

Figure 8: Mean Average Precision.

For comparison purposes, we trained an SSD mobilenet v1 coco model for testing our CCLDataset. SSD is a single shot multibox detector that uses a single deep neural network. When trained on our CCLDataset, the model gave a mAP of 0.673 (67.3\%), which is relatively low compared to the Faster R-CNN inceptionV2 coco model.

\section{Conclusion and Future Work}

This paper performed object detection on cotton leaf images collected in Pakistan to detect diseases on leaves in field condi- tions. In Pakistan the main source of economy is agriculture, particularly cotton crops. This is why we are interested in designing, constructing, and implementing an efficient system to detect and classify cotton leaf diseases, working in real-time. This system can support farmers and learners in diagnosing diseases correctly and beneficial for crops harvested during a single season or a limited period every year. To increase the yield and correctly identifying the leaf diseases of cotton, machine and deep learning is used to train an object detection algorithm on a dataset collected. For this, firstly, we trained the PlantVillage dataset, which contained leaves of fruits and vegetables in laboratory conditions having 38 classes and 53,925 images with a grey background. Different models are used to find the best feature extractor that could be applied as a base model in object detection, which are VGG-16, inceptionV1, and V2. InceptionV2 extracted the best features from the dataset. As a base model, inceptionV2 is selected in object detection using Faster R-CNN on CCLDataset, which contained 700+ cotton leaf field images with four different classes out of which three belong to disease, and one belongs to healthy leaves. Transfer learning played a vital role in training CCLDataset because of the low quantity of images. The loss on this dataset calculated is 0.01275 , while the mean average precision (mAP) is 0.871 or $87.1 \%$.

For future work, a significant contribution could be to increase the number of different plant's datasets to increase farmers and learners support to detect in real-time diverse health and diseases on plants automatically.

\section{Conflict of Interest}

There is no conflict of interest.

\section{Bibliography}

1. Shuli F., et al. "Overview of the cotton in Pakistan and its future prospects". Pakistan Journal of Agricultural Research 31.4 (2018): 396.

2. Wasti SE., et al. "Pakistan Economic Survey 2018-19". Finance Division Government of Pakistan (2019): 11-33.

3. Ahmad I., et al. "Pakistan Economic Survey 2019-2020". Finance Division Government of Pakistan (2020): 17-41

4. Rehman A., et al. "Economic perspectives of major field crops of Pakistan: An empirical study". Pacific Science Review B: Humanities and Social Sciences 1.3 (2015): 145-158. 
5. Khan RA., et al. "Prevalence of the major pests in transgenic and non-transgenic cotton genotype in response to climatic factors and their impact on yield". Archives of Phytopathology and Plant Protection 51.17-18 (2018): 927-938.

6. Hatcher WG and Wei Y. "A survey of deep learning: platforms, applications and emerging research trends". IEEE Access 6 (2018): 24411-24432.

7. O'Shea K and Ryan N. "An introduction to convolutional neural networks". arXiv preprint arXiv:1511.08458 (2015).

8. Rothe PR and Kshirsagar RV. "Cotton leaf disease identification using pattern recognition techniques". 2015 International conference on pervasive computing (ICPC). IEEE (2015): 1-6.

9. Patki SS and Sable GS. "Cotton leaf disease detection \& classification using multi SVM". International Journal of Advanced Research in Computer and Communication Engineering 5.10 (2016): 165-168.

10. Sarangdhar AA and Pawar VR. "Machine learning regression technique for cotton leaf disease detection and controlling using IoT". 2017 International conference of Electronics, Communication and Aerospace Technology (ICECA). IEEE 2 (2017): 449-454.

11. Dubey YK., et al. "Superpixel based roughness measure for cotton leaf diseases detection and classification". 2018 4th International Conference on Recent Advances in Information Technology (RAIT). IEEE (2018): 1-5.

12. Kumari CU., et al. "Leaf disease detection: feature extraction with K-means clustering and classification with ANN". 2019 3rd International Conference on Computing Methodologies and Communication (ICCMC). IEEE (2019): 1095-1098.

13. Khairnar K and Goje N. "Image processing based approach for diseases detection and diagnosis on cotton plant leaf". TechnoSocietal Springer, Cham (2020): 55-65.

14. Patil B and Barkpalli V. "A Perspective View of Cotton Leaf Image Classification Using Machine Learning Algorithms Using WEKA" (2021).

15. Caldeira RF., et al. "Identification of Cotton Leaf Lesions Using Deep Learning Techniques”. Sensors (2021): 3169.

16. Ren S., et al. "Faster R-CNN: towards real-time object detection with region proposal networks". IEEE transactions on pattern analysis and machine intelligence 39.6 (2016): 1137-1149.

17. Weiss K. "A survey of transfer learning". Journal of Big Data 3.1 (2016): 1-40.
Volume 5 Issue 10 October 2021

(C) All rights are reserved by Rehan Sarwar., et al. 